\title{
Erratum: A comparison of
} postoperative emergence agitation between sevoflurane and thiopental anesthesia induction in pediatric patients (Korean J Anesthesiol 2015 Aug; 68(4): 373-378)

\author{
Ji-Seon Son, Eunjoo Jang, Min Wook Oh, Ji-Hye Lee, Young Jin Han and \\ Seonghoon Ko \\ Department of Anesthesiology and Pain Medicine, Chonbuk National University Medical School and Hospital, \\ Jeonju, Korea
}

The article by Son J, et al. entitled, "A comparison of postoperative emergence agitation between sevoflurane and thiopental anesthesia induction in pediatric patients" (Korean J Anesthesiol 2015 Aug; 68(4): 373-378) contained an error in Figure and Figure legend.

Before correction:

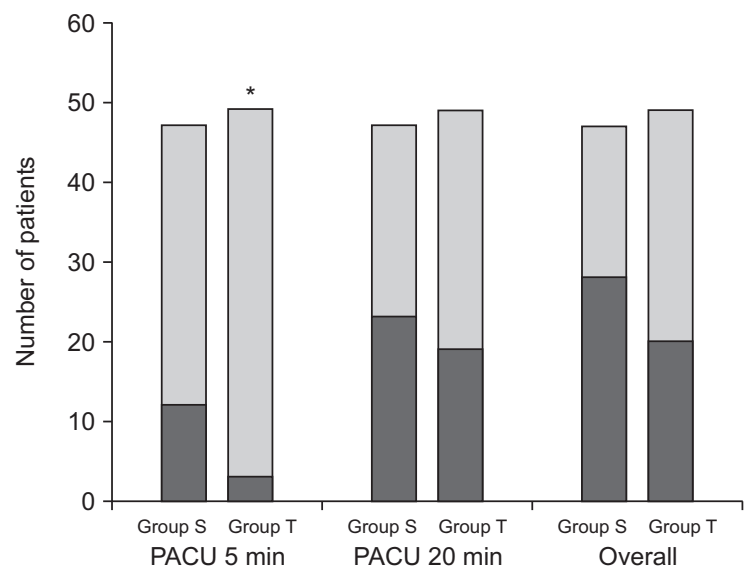

Fig. 2. There was a significant difference in the incidence of emergence agitation (EA) between the two groups at $5 \mathrm{~min}$ after arrival in the postanesthesia care unit (PACU) ( 3 vs. 12 patients, $* \mathrm{P}=0.019)$. However, there were no differences at $20 \mathrm{~min}(23$ vs. 19 patients, $\mathrm{P}=0.425)$ or in the overall incidence $(P=0.102)$. The black stack presents the number of patients who showed EA. Group S: sevoflurane inhalation anesthesia induction group, Group T: intravenous thiopental anesthesia induction group.
The correct information is found below:

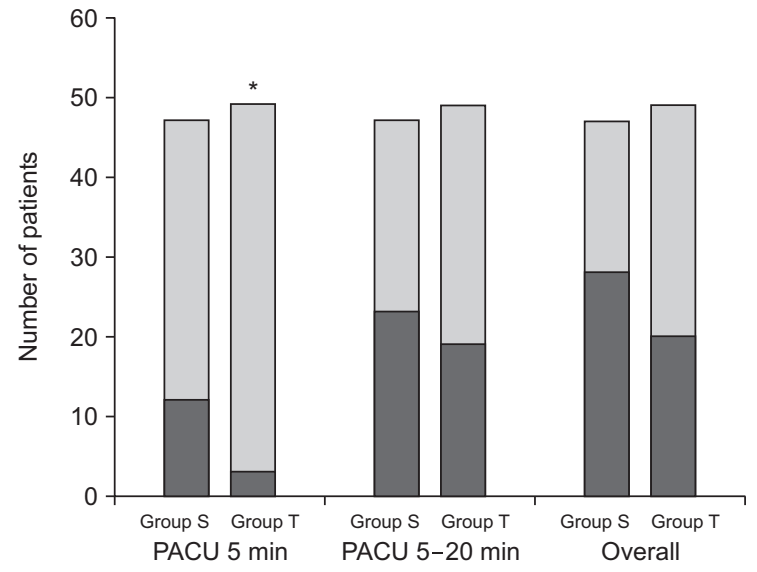

Fig. 2. There was a significant difference in the incidence of emergence agitation (EA) between the two groups at $5 \mathrm{~min}$ after arrival in the postanesthesia care unit (PACU) ( 3 vs. 12 patients, $* \mathrm{P}=0.019)$. However, there were no differences at $5-20 \min (23$ vs. 19 patients, $\mathrm{P}=0.425)$ or in the overall incidence $(P=0.102)$. The black stack presents the number of patients who showed EA. Group S: sevoflurane inhalation anesthesia induction group, Group T: intravenous thiopental anesthesia induction group.

PACU times are erroneously given as $20 \mathrm{~min}$; instead it should read as 5-20 min.

The authors apologize for any inconvenience this mistake may have caused.

Korean J Anesthesiol 2016 February 69(1): 100

http://dx.doi.org/10.4097/kjae.2016.69.1.100

(c) This is an open-access article distributed under the terms of the Creative Commons Attribution Non-Commercial License (http://creativecommons.org/ licenses/by-nc/4.0/), which permits unrestricted non-commercial use, distribution, and reproduction in any medium, provided the original work is properly cited. 